\title{
Metabolomics Approach for Hazard Identification in Human Health Assessment of Environmental Chemicals
}

\author{
Suryanarayana V. Vulimiri ${ }^{1}$, Brian Pachkowski², \\ Ambuja S. Bale ${ }^{1}$ and Babasaheb Sonawane ${ }^{1}$ \\ ${ }^{1}$ U.S. Environmental Protection Agency, Office of Research and Development, \\ ${ }^{2}$ Oak Ridge Institute for Science and Education Postdoctoral Fellow, \\ National Center for Environmental Assessment, Washington DC, \\ USA
}

\section{Introduction}

Exposure to xenobiotics induces complex biochemical responses in mammalian cells resulting in several perturbations in cellular toxicity pathways. Within the context of systems biology, such biochemical perturbations can be studied individually using "omics" approaches such as toxicogenomics, transcriptomics, proteomics and metabolomics (Heijne et al., 2005). The objective of this chapter is to examine how the metabolomics approach can be used in identifying the risk posed by environmental chemicals to human health using selective examples of organ toxicity. Metabolomics is a medium-to-high throughput technique employing predominantly mass spectrometry (MS) and nuclear magnetic resonance (NMR) technology (Roux et al., 2011) for the identification and characterization of endogenous metabolites of low molecular weight $(<1800 \mathrm{Da})$ arising from different biochemical pathways either as primary or secondary metabolites (Idle \& Gonzalez, 2007). The sum total of all small metabolites is referred to as the "metabolome". Metabolomics has also been applied to the identification of low molecular weight, exogenous metabolites of xenobiotics (Roux et al., 2011; Rubino et al., 2009). With these capabilities, metabolomics represents a relatively quick and informative approach for assessing the physiological response to environmental chemicals.

\section{Human health risk assessment}

Chemicals in the environment could pose potential risks to human health. In order to inform the assessment of risks from chemical exposures, the U.S. National Research Council (NRC) published a report entitled, "Risk Assessment in the Federal Government: Managing the Process," more commonly known as the "Red Book" (NRC, 1983), which has been widely accepted and endorsed by the U.S. Environmental Protection Agency (U.S. EPA) and other federal agencies. This risk assessment process consists of four steps: hazard identification, doseresponse assessment, exposure assessment, and risk characterization. The focus herein is on hazard identification, which has been defined as "identification of the contaminants that are 
suspected to pose health hazards, quantification of the concentrations at which they are present in the environment, a description of the specific forms of toxicity (i.e. neurotoxicity, carcinogenicity, etc.) that can be caused by the contaminants of concern, and an evaluation of the conditions under which these forms of toxicity might be expressed in exposed humans" (NRC, 1994).

For human health assessment of chemicals, non-cancer or cancer risk values are derived based on the selection of a critical endpoint of toxicity or several endpoints (e.g. biochemical, pathological, physiological, and behavioral abnormalities) of adverse health outcomes. Uncertainty factors are applied to the lowest dose associated with the critical health outcome(s) in order to derive the resulting exposure level for non-cancer toxicity. These uncertainty factors attempt to account for exposure duration, pharmacokinetic, and pharmacodynamic data gaps associated with inter- and intra-species extrapolation.

The U.S. EPA and the International Agency for Research on Cancer (IARC) evaluate the evidence for carcinogenesis in humans from epidemiological, experimental animal, and mechanistic data to determine the qualitative cancer classification for humans. In addition, the U.S. EPA evaluates exposure-response relationships and develops quantitative cancer risk values based on the observed tumors that correspond to a unit exposure (U.S. EPA, 2005). Uncertainties with cancer risk values are presented and are generally associated with the mode of action (MOA) for carcinogenicity.

One of the major concerns with cancer risk assessment is false-positive animal tumor findings. Having an understanding of the mechanism(s) leading to carcinogenicity would help in developing a better perspective of whether a carcinogen in experimental animals is likely to be a carcinogen in humans. For example, correlating a metabolomic profile of a suspected carcinogen between human exposures (environmental or occupational) and experimental animal exposure studies would be highly useful. If similar biochemical markers were to appear across the human and animal metabolomic profiles, that information would help in informing similarities or differences in interspecies mechanisms. Further, if the chemical was demonstrated to be a carcinogen in animals through a traditional two-year animal bioassay, but there was inconclusive epidemiological evidence, the similarity in metabolomic data could be used along with other mechanistic data (e.g. mutagenicity/genotoxicity assays, cell proliferation findings, oxidative stress, epigenetics, etc.) to support or refute human carcinogenicity. In this regard, metabolomics information could be used to support mechanistic data to augment the animal and human findings.

\section{The potential of "omics" data to inform mode of action of environmental chemicals}

In developing a human health evaluation for environmental chemical hazard identification, it is ideal to have information on the key mechanistic events leading to an adverse health outcome. In this regard, mode of action (MOA) is an important part of hazard identification. MOA can be defined as "a sequence of key events and processes, starting with interaction of an agent with a cell, proceeding through operational and anatomical changes, and resulting in cancer formation" (U.S. EPA, 2005). A 'key event' is defined as "an empirically observable precursor which is by itself a necessary component of the MOA or is a biologically-based measurable marker for such a component" (U.S. 
EPA, 2005). In vitro systems (e.g. cell cultures) and in vivo models (e.g. experimental animals or human population studies) have identified several of the early key events such as oxidative stress, inflammation, genotoxicity, and cytotoxicity that occur from toxicant exposure. Since metabolomics measures biological response at the molecular level, this approach can identify the metabolites associated with the sequence of 'key events' and the processes inherent to the mechanism(s) of xenobiotic toxicity. The metabolomics approach could generate several mode(s) of action hypotheses using a nontargeted approach. The individual MOA hypothesis thus generated could be tested in targeted approaches (e.g. measuring glutathione reduction from oxidative stress) using more conventional assays. Metabolomics data could be used to further inform the mode(s) of action in experimental animals associated with carcinogenicity or with noncancer health outcomes, which may help to confirm the relevancy of the observations in experimental animals to humans.

\section{Metabolomics approach in investigating environmental chemical exposure}

Environmental chemicals act through multiple toxicity pathways via a multitude of mechanisms (Guyton et al., 2009). To date, very limited toxicogenomics information has been applied to the field of risk assessment (Boverhof \& Zacharewski, 2006; Mortensen \& Euling, 2011). There is a paucity of relevant metabolomic information for application to human health risk assessment of environmental chemicals. To date, the available literature suggests an informative role of metabolomics in understanding the mode of action of environmental xenobiotics (Vulimiri et al., 2011; Vulimiri et al., 2009).

In general, human data are relatively sparse for many environmental chemicals with respect to both non-cancer and cancer health outcomes. Most human data are occupational, where exposure levels are generally higher than those encountered in the environment. In many cases, there are not any environmental or occupational human exposures that could be used to corroborate the animal data. In these cases, animal data are generally used to develop non-cancer and cancer risk values for human health assessments thereby raising issues of uncertainty associated with interspecies extrapolation. Metabolomic data could be used to fill in such data gaps. For example, in vitro metabolomic assays with human cells may be developed to compare with animal metabolomic profiles to determine if there are potentially similar mechanisms of toxicity or for identifying toxicity pathways. As a result, this characterization of biochemical mechanisms of toxicity would inform hazard identification for use in the human health risk assessment process.

\section{Ability of metabolomics to differentiate gender, phenotypic, and genetic differences, and organ-specific effects}

Since metabolomics analyzes endogenous and exogenous (xenobiotic-derived) low molecular weight metabolites, this approach has been applied to the differentiation of metabolic profiles between phenotypes and genotypes. As briefly discussed below, metabolomics has the ability to inform gender, genetic, and phenotypic differences as well as organ-specific effects. For understanding the toxicity of environmental agents, the utility of such information would clarify toxicodynamic uncertainty associated with the extrapolation between species as well as within species (i.e. human) variability. 


\subsection{Gender differences}

Metabolomics approach can differentiate between gender-dependent differences in the metabolic profiles of untreated or control experimental animals. In a study aimed at the identification of novel biomarkers of effect using chemicals with diverse modes of action, one research group (van Ravenzwaay et al., 2007) pooled the metabolomic data (i.e. plasma profiles) from eleven individual experiments involving 670 male and female control Wistar rats over a span of one year. After principal component analysis (PCA), the authors found that the metabolic profiles were clustered into separate groups for males and females suggesting a stable metabolome of the control rats during the study period. This observation highlights the ability of metabolomics to potentially identify unique gender responses to chemical exposure.

\subsection{Genetic differences and phenotypic effects}

The metabolomics approach can be further used for studying the relationship between the genotype and phenotype of the organism. Genetic polymorphisms in human genes are known to modify exposure to environmental health hazards and are a source of uncertainty when assessing risk from environmental chemicals (Ginsberg et al., 2009; Kelada et al., 2003). Genetic differences have been shown to reflect changes in the metabolite profiles of individuals. In a human population, genetically determined variants (e.g. those associated with fatty acid metabolism) in metabolic phenotype (metabotype) have been identified by simultaneously detecting single-nucleotide polymorphisms (SNPs) in a genome-wide association study (GWAS) and endogenous serum metabolites (Gieger et al., 2008).

This is also evident in the field of functional genomics where a change in phenotype is observed due to gene-related alterations (reverse-genetics) such as deletions or insertions leading to silent mutations as in yeasts (Raamsdonk et al., 2001). Specific gene mutations can also be evaluated using metabolic footprinting (Szeto et al., 2010). Such metabolomic data could provide basic information regarding gene product function, particularly in the context of environmental exposure.

Also, the genotypes of animals, as in genetically manipulated animal models (e.g. gene knockout and transgenic mice), have been used effectively for understanding the metabolism of toxicants mediated by cytochrome P450 (CYP) isozymes, in order to further elucidate mechanisms of toxicity. For example, MS-based approaches were able to distinguish between the metabolic profiles for Cyp2e1-null, CYP1A2-humanized, and wildtype mice after exposure to the ubiquitous dietary carcinogen 2-amino-1-methyl-6phenylimidazo[4,5-b]pyridine (Chen et al., 2007) or the hepatotoxic agent acetaminophen (Chen et al., 2008). As a result, the metabolomics approach could be used to identify mechanistic changes stemming from genetic differences.

\subsection{Organ-specific effects}

Metabolomics approach has been utilized in identifying specific profiles that are altered in different organs in response to toxicant injury. The following represents a brief discussion of three select organ toxicities associated with exposure to a given chemical or mixture of 
chemicals. The extent of the metabolomic datasets varies for each type of organ toxicity; however, discussion focuses on how metabolomic investigations have contributed to some understanding of mechanisms of toxicity. Some of these metabolic changes are specific but many are nonspecific for the selected organ toxicities.

\subsubsection{Hepatotoxicity (Carbon tetrachloride)}

Carbon tetrachloride $\left(\mathrm{CCl}_{4}\right)$ is a well-studied hepatotoxicant and carcinogen that has been used as a fumigant (Manibusan et al., 2007). The mechanisms underlying $\mathrm{CCl}_{4}$ toxic responses are fairly well understood. In rats, $\mathrm{CCl}_{4}$ exposure results in mild to severe centrilobular necrosis of the liver with elevation of serum alanine aminotransferase (ALT) and aspartate aminotransferase (AST) activities suggestive of hepatotoxicity. In addition, $\mathrm{CCl}_{4}$ induces lipid peroxidation, oxidative stress, and regenerative proliferation, eventually leading to hepatocarcinogenesis. The following brief discussion focuses on how metabolomics has been used to identify metabolites associated with these known mechanisms of toxic response.

Metabolomic analyses have identified several aldehydes (e.g. formaldehyde, acetaldehyde, propanal, butanal, pentanal, hexanal and malondialdehyde) excreted in urine (De Zwart et al., 1997) or in exhaled breath (Gee et al., 1981) from rodents dosed with $\mathrm{CCl}_{4}$. Similarly, short-chain hydrocarbons (e.g. pentane and ethane) generated during lipid peroxidation have been reported in the breath of humans suggestive of $\mathrm{CCl}_{4}$-induced oxidative stress (Hwang \& Kim, 2007). These reactive aldehydes arising from lipid peroxidation have been shown to be quenched by reduced glutathione (GSH), causing GSH depletion which leads to cirrhosis in the liver of rats (Cabre et al., 2000). Further, GSH depletion causes a perturbation of prooxidant-antioxidant balance eventually leading to oxidative stress (Ichi et al., 2009). Metabolomic studies have provided support that oxidative stress induced by $\mathrm{CCl}_{4}$ generates reactive oxygen species (ROS) capable of causing DNA damage as shown by an increase in 8-oxodeoxyguanosine (8-oxodG) and malondialdehyde-deoxyguanosine $\left(\mathrm{M}_{1} \mathrm{G}\right)$ adducts (Beddowes et al., 2003). Also, lipid peroxidation products, such as 4-hydroxynonenal, can form etheno adducts, which are capable of inducing mutations in critical genes through base-pair substitutions (Barbin, 2000; Chung et al., 1999).

Further, $\mathrm{CCl}_{4}$-induced oxidative stress inhibits energy metabolism as observed by changes in levels of Krebs cycle intermediates (i.e. citric acid, 2-oxoglutarate, and succinate), which has an overall effect on glutathione metabolism eventually leading to hepatotoxicity (Huang et al., 2008; Kim et al., 2008; Lin et al., 2009; Robertson et al., 2000). These earlier cellular perturbations further affect $\mathrm{Ca}^{+2}$ homeostasis, cause protein and phospholipid degradation, and potentially lead to cytotoxicity (Manibusan et al., 2007), apoptosis and necrosis of the liver tissue (Sun et al., 2001). Furthermore, membrane phospholipid degradation leads to the release of high levels of $o$-phosphoethanolamine, a well-known biomarker of cytotoxicity that can be detected using a metabolomic approach (Wang et al., 2009).

$\mathrm{CCl}_{4}$ toxicity also leads to an inflammatory response characterized by the release of arachidonic acid, which is the precursor for a cascade of events leading to prostaglandin and leukotriene synthesis involving cyclooxygenases and lipoxygenases, respectively (Basu, 2003), and other newer inflammatory biomarkers, such as resolvins, protectins, and maresins (Liu et al., 2009; Serhan, 2009). Following cytotoxicity, liver tissue undergoes 
regenerative proliferation and activation of the urea cycle characterized by polyamine biosynthesis, the latter being a hallmark of cellular proliferation and differentiation (Heby, 1981). Detection of increased levels of urea cycle metabolites such as putrescine, ornithine, spermine, and spermidine (Pegg et al., 1981) in $\mathrm{CCl}_{4}$-exposed rats by metabolomics approach gives an early indication of regenerative cell proliferation, which has been detected at a later stage of disease progression using conventional toxicity assays.

Overall, metabolomics studies have identified important biomarkers for $\mathrm{CCl}_{4}$-induced toxicity including: aldehydes (lipid peroxidation); GSH/GSSG ratio (oxidative stress); 8oxodG, $\mathrm{M}_{1} \mathrm{G}$, and etheno adducts (genotoxicity); o-phosphoethanolamine (cytotoxicity); arachidonic acid and prostaglandins (inflammation); and polyamines (regenerative cell proliferation), which are also observed in conventional assays (Vulimiri et al., 2011). This brief discussion further supports the use of metabolomics for investigating toxicity associated with environmentally related chemicals.

\subsubsection{Neurotoxicity (Methylmercury)}

Methylmercury is a chemical that was originally used as a fungicide for agricultural purposes in the early 20th century. In one of the earliest studies of methylmercury toxicity, occupational exposure to this chemical resulted in detriments in neurological functions in factory workers and changes in brain morphology with specificity to the cerebellar area. Additionally, in one of the most noted disasters in the $20^{\text {th }}$ century, methylmercury exposure through consumption of fish was determined to be the causative agent for producing neurological deficits in fishermen and neurodevelopmental abnormalities in children in Minamata Bay, Japan (Ekino et al., 2007).

Traditional approaches in hazard characterization have concluded that the most sensitive (critical) effect observed from methylmercury exposure is neurobehavioral changes in children [as reviewed in (U.S. EPA, 2005) and Agency for Toxic Substances and Disease Registry (ATSDR, 1999)]. An uncertainty that was highlighted in both of these human health assessment evaluations was that of pharmacodynamic variability in the human population. One approach to address pharmacodynamic variability is to have an understanding of the key mechanism(s) that results in the sensitive effect. There have been several reviews on the neurological mechanisms associated with methylmercury (Ekino et al., 2007; Myers et al., 2009). One of the primary mechanisms associated with methylmercury neurotoxicity in both the adult and developing brain is cytotoxicity. Methylmercury has been demonstrated to induce oxidative stress in the nervous system. Additionally, in vitro studies have reported that methylmercury treatment results in apoptosis in glial cells, cerebellar granule cells, and in neuronal cell lines such as differentiated PC12 cells and neuroblastoma cells. Methylmercury exposure in rats has also resulted in apoptosis in the cerebellum as well as loss of astrocytes in monkeys. The cell death pathway for methylmercury has been highly characterized as reviewed recently (Ceccatelli et al., 2010). In summary, several studies have demonstrated that methylmercury induces caspase-dependent apoptosis in primary neuronal cell cultures and cell lines.

Metabolomic analysis for methylmercury was conducted using an in vitro methodology proposed by the European Commission Joint Research Centre in 2008 (van Vliet et al., 2008). Dynamic reaggregating neuronal cultures prepared from 16-day old rat fetal telencephalon 
were used for this analysis. The identified putative biomarkers were $\gamma$-aminobutyric acid (GABA), choline, glutamine, creatine, and spermine. The authors were able to speculate potential mechanisms of methylmercury from results of traditional biochemical assays (Eskes et al., 2002; Monnet-Tschudi et al., 1996) where the enzymatic activities of neuronal enzymes including glutamine synthesis, choline acetyltransferase, and glutamic acid decarboxylase were reported to be significantly decreased. In essence, the decreased levels of GABA and choline in the in vitro metabolomic study may help to explain these decreases in enzymatic activity which then leads to neuron-specific toxicity. This information correlates to the observed increases in apoptotic cell death associated with methylmercury. Similarly, the increased creatine levels that were reported from methylmercury treatment were correlated to gliosis (proliferation of astrocytes in the central nervous system) by the authors. Creatine is generally linked to brain osmoregulation (Bothwell et al., 2001) and increased creatine levels would lead to increased activity of glial cells that could result in gliosis.

Thus, a very limited metabolomic dataset for methylmercury adds to the mechanistic profile of this compound and helps establish a temporality of the key events leading to cytotoxicity in the brain. In the case of using the metabolomic data for methylmercury, the observed changes in the neuronal metabolites provide supportive, early evidence of later stage events leading to brain cytotoxicity. Metabolomic data from in vivo studies or from incidental human exposure would demonstrate any pharmacodynamic differences and if so, how such differences could be quantified through the available data rather than the default use of uncertainty factors.

\subsubsection{Pulmonary toxicity (Cigarette smoke)}

Cigarette smoking is one of the major etiological reasons for pulmonary toxicity and lung cancer. Cigarette smoke contain at least 4000 chemicals from different chemical classes, of which at least 60 are well-established carcinogens in experimental animals (Hecht, 2006). Cigarette smoke has been shown to induce oxidative stress and an inflammatory response in lungs, and chronic exposure is known to cause cancer. While cigarette smoke is most deleterious to the smoker, environmental or passive exposure to smoke (i.e. second-hand smoke) can lead to adverse health effects to bystanders (U.S. EPA, 1992). Using the metabolomics approach in A549 human lung epithelial cells it has been shown that several biochemical pathways are altered by either the whole smoke (WS) or its component phases i.e. wet total particulate matter (WTPM) or gas/vapor phase (GVP) (Vulimiri et al., 2009). Exposures to the different phases lead to unique biochemical alterations in A549 cells. For example, WTPM exposure resulted in changes in the Krebs cycle and urea cycle metabolites, whereas GVP exposure resulted in alterations in hexosamines and in lipid metabolism. These investigators found that exposure to cigarette smoke was associated with perturbations in the metabolism of glutathione, amino acids, lipids, and nucleotides. Alterations were also seen in the urea cycle, Krebs cycle, and the production of polyamines and cellular cofactors.

Oxidative stress is an important consequence associated with cigarette smoke. The oxidants produced or found in cigarette smoke can react with and damage cellular macromolecules including proteins, DNA, and lipid membranes. Additionally, these oxidants can act as signaling molecules that can influence cell proliferation (Faux et al., 2009). However, cells 
contain antioxidant defenses, such as GSH to prevent oxidative damage (Rahman \& MacNee, 1999). The metabolomics approach has indeed found changes in metabolites associated with these effects of cigarette smoke. Predominant changes in metabolites from exposure to WS included glutathione and $\gamma$-glutamylglutamine, which showed 51 and 13fold increases compared to control cells. The increased levels of metabolites within the glutathione pathway (i.e. glutathione and $\gamma$-glutamylglutamine) suggest a protective response against oxidative stress, which can result from WS. These data correlate with human microarray data that demonstrated an antioxidant response to cigarette smoke through the induction of genes associated with glutathione metabolism (Spira et al., 2004). Further, Vulimiri and colleagues (Vulimiri et al., 2009) observed increased levels (16.4-fold) of $o$-phosphoethanolamine, a marker of phospholipid degradation that may indicate cell membrane damage. Additional observations included increased arachidonic acid levels that may suggest an inflammatory response and markers (e.g. putrescine) of cell proliferation.

Conversely, there was a statistically significant decrease in glutathione levels from exposure to WTPM and GVP when compared to controls. Predominant metabolite changes in these phases were acetylcarnitine (4.5-fold increase) and palmitoleate (5.2-fold increase) for WTPM and GVP, respectively, both of which indicated changes in lipid metabolism (Vulimiri et al., 2009). In summary, these authors (Vulimiri et al., 2009) demonstrated how metabolomics could differentiate metabolic responses caused by exposure to complex mixtures (i.e. cigarette smoke) and also provided empirical data for metabolic changes for known markers of toxicity (e.g. decreased GSH, membrane damage) associated with exposure to cigarette smoke.

\section{Integrating "omics" data into risk assessment}

The field of toxicology is advancing with several of the high throughput screening techniques taking major roles in understanding toxicity pathways. More specifically toxicogenomics and proteomics have contributed to identifying the mechanisms of toxicity pathways (Burchiel et al., 2001; Heijne et al., 2005). Integration of data from different "omics" approaches would help clarify the mode of action of xenobiotics especially at low dose levels, which are relevant to environmental exposures. Thus, combining "omic" techniques such as toxicogenomics, transcriptomics, proteomics, and metabolomics promises to provide a robust understanding of biological responses to xenobiotics. Under this paradigm, effects on genes and their downstream products, namely proteins and the metabolites produced by proteins, can be assessed together. Researchers have utilized diverse "omics" approaches to understand the mode(s) of action or mechanism of different toxicity pathways in various experimental models. Some studies have indeed shown a good correlation between the genomics and metabolomics approaches. For example, metabolomics approach has demonstrated alterations in the pathways associated with lipid peroxidation, DNA damage and repair, and cell proliferation in rats exposed to $\mathrm{CCl}_{4}$ which was consistent with the expression of transcripts associated with steatosis/fibrosis that were specific to cell injury and regeneration (Chung et al., 2005a; Chung et al., 2005b; Fountoulakis et al., 2002). Additionally, metabolomics has identified biochemical alterations in pathways associated with oxidative stress, inflammation, cell proliferation, and cytotoxicity in human lung epithelial cells exposed to cigarette smoke (Vulimiri et al., 2009). These observations are supported by data from genomic (Harvey et al., 2007; Meng et al., 
2006; Spira et al., 2004) and proteomic (Carter et al., 2011; Kelsen et al., 2008; Zhang et al., 2008) investigations that also detected markers associated with the pathways affected by exposure to cigarette smoke.

Conversely, the integration of "omics" technologies has also demonstrated some limitations. For example, a study by Steiling and co-workers found a discrepancy between genomic and proteomic data. Specifically, gel electrophoresis followed by LC-MS analysis identified 41 proteins whose expression would not have been detected by microarray analysis (Steiling et al., 2009). Such data highlight the importance of assessing more downstream markers (i.e. proteins or metabolites) that may provide a more accurate understanding of the biological responses to chemical exposures.

\section{Shift in the risk assessment paradigm from apical endpoints to biochemical perturbations}

Historically, human health risk assessment relied on identifying apical endpoints, defined as "observable outcomes in a whole organism, such as a clinical sign or pathologic state, indicative of a disease state that can result from exposure to a toxicant", such as birth defects, neurologic deficits, and tumor number that are obtained from high-dose animal bioassays (Krewski et al., 2010). However, the mechanistic relevance of these data can be tenuous considering the need for interspecies extrapolation and that environmental exposures may be orders of magnitude lower. Recently, the NRC report on "Toxicity Testing in the 21st Century: A Vision and Strategy" suggested a fundamental change in the risk assessment paradigm where the reliance on apical endpoints of toxicity would be replaced in favor of in vitro toxicity testing for identifying perturbations in biochemical pathways. Such an approach would employ cell lines, particularly human-based lines, and high throughput screening assays (e.g. genomics, proteomics) with computational toxicology methods. As a result, this approach would also lead to an overall increase in the efficiency of toxicity testing and decrease in animal usage [as reviewed in (Krewski et al., 2010)]. As pointed out by the NRC committee, the use of these low-dose, human-based in vitro systems would negate concerns associated with high-dose, animal data. Since metabolomics can identify early perturbations in biochemical pathways, this technology is poised to become an important element of this proposed risk assessment paradigm.

\section{Advantages of metabolomics approach for environmental chemical assessment}

Metabolomics approach offers several advantages for understanding the mechanisms of toxicity of environmental chemicals and informing human health assessments. One advantage of the metabolomics approach is the relatively non-invasive (e.g. urine samples) nature of this technique. In this context, samples from humans subjected to an incidental environmental exposure can be easily collected, analyzed, and correlated to metabolomic profiles from animal studies in order to identify critical effects from the exposure. Aside from quantitative differences, metabolic pathways are evolutionally conserved across different species; metabolomics data can be qualitatively extrapolated or interpreted at the molecular level among and between species. Unlike genomics and proteomics, metabolomics databases offer information on the structural, physicochemical, 
pharmacological and spectral profiles as well as biological functions of metabolites (Go, 2010). From a technical standpoint, sample preparation is relatively minimal for metabolomic analyses compared to genomics and proteomics approaches. Also, since metabolomics allows for the a priori selection of metabolites, the investigator has better control over the number of standards that need to be procured or synthesized for analysis.

A survey of the available literature already demonstrates the value of using metabolomics for elucidating the mode of action of environmental chemicals. Since a number of matrices are amenable to metabolomic analysis, various systems including in vitro and in vivo models can be employed to identify metabolic perturbations associated with exposure to environmental chemicals. Some of the mammalian in vitro systems involved exposing A549 human lung carcinoma cells to cigarette smoke (Vulimiri et al., 2009), human embryonic stem cells to various teratogens (West et al., 2010), and reaggregating neuronal cultures to methylmercury (van Vliet et al., 2008). From in vivo studies, intact organs from exposed experimental animals have been evaluated to include the analysis of intact lung after intratracheal instillation of silica dust (Hu et al., 2008) as well as liver and lung after exposure to 1-nitronaphthalene (Azmi et al., 2005). Also, metabolomics has been able to demonstrate different metabolic responses between mixtures of environmental chemicals. For example, in assessing the inflammatory response, Schmelzer et al (2006) found unique lipid profiles between rats exposed to 1nitronaphthalene and those exposed to a mixture of 1-nitronaphthalene and ozone. As highlighted earlier in this chapter, metabolomics approach has been shown to differentiate the metabolic changes attributed to the complex mixtures of whole smoke versus its two constituent physical phases (Vulimiri et al., 2009).

\section{Limitations of metabolomics research}

Regardless of whether a chemical being investigated is a pharmaceutical or environmental pollutant, a critique of metabolomics has been the detection of metabolites that appear to be changed independent of the toxicant, its mode of action, and target tissue. Such common metabolites were previously termed the "usual suspects" by Robertson and often include 2-oxoglutarate, citrate, succinate, and trimethyl amine/trimethyl amine oxide (Robertson, 2005). From a data interpretation standpoint, these "usual suspects" may confound the identification of metabolites that have true value as specific markers of organ toxicity by skewing results from pattern separation analyses (Connor et al., 2004; Robertson, 2005). Connor et al (2004) found that urinary metabolite changes, including many of the "usual suspect" metabolites, can be associated with systemic or secondary toxicity effects, namely reductions in food intake and body weight. Additionally, the authors suggest that such metabolites may still inform mechanisms of toxicity when put into the context of diet and weight change. Conversely, the authors did identify exposures where common metabolites were specific for toxicant exposure. For example, succinate and 2-oxoglutarate were specifically associated with exposure to the hepatotoxicant anaphthylisothiocyanate, rather than resulting from secondary toxicity (Connor et al., 2004). Research by other investigators has identified metabolites as novel biomarkers associated with specific diseases and for specific toxicities. For example, 5-oxoproline has been demonstrated to be a specific marker of bromobenzene-induced hepatotoxicity (Waters et al., 2006). 


\section{Future directions/research needs}

A goal of this chapter has been to highlight how metabolomics approach can be used to better understand the toxicity of environmental chemicals, with a particular focus on hazard identification and mode of action. However, due to its relative infancy compared to conventional toxicity assays and other "omic" technologies, metabolomic information for environmental chemicals is sparse. As interest in metabolomics increases and as this approach becomes more accessible to researchers focused on environmental chemicals, this database will grow. A contemporary issue regarding xenobiotics is the influence of the microbiome on immunity, metabolism, and human health [reviewed in (Han et al., 2010)]. This microbiome involves the sum of all the microorganisms (e.g. bacteria) that internally and externally reside on an individual or animal. Of these microorganisms, the gut microbiota has been shown to influence the metabolism of several xenobiotics. Accordingly, the microbiome is likely to be important in the toxicity of environmental chemicals and pertinent to human health assessments (Betts, 2011). Thus, the influence of the microbiome is an important aspect of chemical toxicity that should be further studied using metabolomics.

\section{Conclusions}

Metabolomics is an emerging medium-to-high throughput technique which measures the endogenous biochemicals affecting different metabolic pathways and can be useful in characterizing the hazards of environmental chemicals. Identifying metabolic perturbations caused in mammalian cell systems following chemical exposure helps in elucidating the predominant toxicity pathways. Some of the advantages of using metabolomic data for hazard identification - one of the key steps in risk assessment-include the ability to inform gender, genetic, and organ-specific effects in a relatively expedient manner. As briefly discussed, metabolomics can identify early biochemical perturbations associated with toxicity in the hepatic, nervous, and pulmonary systems caused by selected environmental chemicals. As surveyed, various research systems using metabolomics demonstrate how metabolomic data could be used for hazard identification and mode of action characterization for environmental chemicals. Overall, metabolomics represents an opportunity to develop a better understanding of the toxicity of environmental chemicals and could further impact the human health assessment of these chemicals.

\section{Disclaimer}

The views expressed in this chapter are those of the authors and do not necessarily represent the views or policies of the U.S. Environmental Protection Agency.

\section{References}

ATSDR (1999). Toxicological profile for mercury, Agency for Toxic Substances and Disease Registry, Atlanta, GA, Available from: http://www.atsdr.cdc.gov/toxprofiles/tp46.pdf

Azmi, J.; Connelly, J.; Holmes, E.; Nicholson, J.K.; Shore, R.F. \& Griffin, J.L. (2005). Characterization of the biochemical effects of 1-nitronaphthalene in rats using 
global metabolic profiling by NMR spectroscopy and pattern recognition, Biomarkers, Vol. 10, No. 6, pp. 401-16.

Barbin, A. (2000). Etheno-adduct-forming chemicals: from mutagenicity testing to tumor mutation spectra, Mutat Res, Vol. 462, No. 2-3, pp. 55-69.

Basu, S. (2003). Carbon tetrachloride-induced lipid peroxidation: eicosanoid formation and their regulation by antioxidant nutrients, Toxicology, Vol. 189, No. 1-2, pp. 113-27.

Beddowes, E.J.; Faux, S.P. \& Chipman, J.K. (2003). Chloroform, carbon tetrachloride and glutathione depletion induce secondary genotoxicity in liver cells via oxidative stress, Toxicology, Vol. 187, No. 2-3, pp. 101-15.

Betts, K.S. (2011). A study in balance: how microbiomes are changing the shape of environmental health, Environ Health Perspect, Vol. 119, No. 8, pp. a340-6.

Bothwell, J.H.; Rae, C.; Dixon, R.M.; Styles, P. \& Bhakoo, K.K. (2001). Hypo-osmotic swelling-activated release of organic osmolytes in brain slices: implications for brain oedema in vivo, J Neurochem, Vol. 77, No. 6, pp. 1632-40.

Boverhof, D.R. \& Zacharewski, T.R. (2006). Toxicogenomics in risk assessment: applications and needs, Toxicol Sci, Vol. 89, No. 2, pp. 352-60.

Burchiel, S.W.; Knall, C.M.; Davis, J.W., 2nd; Paules, R.S.; Boggs, S.E. \& Afshari, C.A. (2001). Analysis of genetic and epigenetic mechanisms of toxicity: potential roles of toxicogenomics and proteomics in toxicology, Toxicol Sci, Vol. 59, No. 2, pp. 193-5.

Cabre, M.; Camps, J.; Paternain, J.L.; Ferre, N. \& Joven, J. (2000). Time-course of changes in hepatic lipid peroxidation and glutathione metabolism in rats with carbon tetrachloride-induced cirrhosis, Clin Exp Pharmacol Physiol, Vol. 27, No. 9, pp. 694-9.

Carter, C.A.; Misra, M. \& Pelech, S. (2011). Proteomic Analyses of Lung Lysates from ShortTerm Exposure of Fischer 344 Rats to Cigarette Smoke, J Proteome Res, Vol. (In Press), No.

Ceccatelli, S.; Dare, E. \& Moors, M. (2010). Methylmercury-induced neurotoxicity and apoptosis, Chem Biol Interact, Vol. 188, No. 2, pp. 301-8.

Chen, C.; Krausz, K.W.; Idle, J.R. \& Gonzalez, F.J. (2008). Identification of novel toxicityassociated metabolites by metabolomics and mass isotopomer analysis of acetaminophen metabolism in wild-type and Cyp2e1-null mice, J Biol Chem, Vol. 283, No. 8, pp. 4543-59.

Chen, C.; Ma, X.; Malfatti, M.A.; Krausz, K.W.; Kimura, S.; Felton, J.S.; Idle, J.R. \& Gonzalez, F.J. (2007). A comprehensive investigation of 2-amino-1-methyl-6phenylimidazo[4,5-b]pyridine (PhIP) metabolism in the mouse using a multivariate data analysis approach, Chem Res Toxicol, Vol. 20, No. 3, pp. 531-42.

Chung, F.L.; Nath, R.G.; Nagao, M.; Nishikawa, A.; Zhou, G.D. \& Randerath, K. (1999). Endogenous formation and significance of 1,N2-propanodeoxyguanosine adducts, Mutat Res, Vol. 424, No. 1-2, pp. 71-81.

Chung, H.; Hong, D.P.; Jung, J.Y.; Kim, H.J.; Jang, K.S.; Sheen, Y.Y.; Ahn, J.I.; Lee, Y.S. \& Kong, G. (2005a). Comprehensive analysis of differential gene expression profiles on carbon tetrachloride-induced rat liver injury and regeneration, Toxicol Appl Pharmacol, Vol. 206, No. 1, pp. 27-42.

Chung, H.; Hong, D.P.; Kim, H.J.; Jang, K.S.; Shin, D.M.; Ahn, J.I.; Lee, Y.S. \& Kong, G. (2005b). Differential gene expression profiles in the steatosis/fibrosis model of rat liver by chronic administration of carbon tetrachloride, Toxicol Appl Pharmacol, Vol. 208, No. 3, pp. 242-54.

Connor, S.C.; Wu, W.; Sweatman, B.C.; Manini, J.; Haselden, J.N.; Crowther, D.J. \& Waterfield, C.J. (2004). Effects of feeding and body weight loss on the 1H-NMR- 
based urine metabolic profiles of male Wistar Han rats: implications for biomarker discovery, Biomarkers, Vol. 9, No. 2, pp. 156-79.

De Zwart, L.L.; Venhorst, J.; Groot, M.; Commandeur, J.N.; Hermanns, R.C.; Meerman, J.H.; Van Baar, B.L. \& Vermeulen, N.P. (1997). Simultaneous determination of eight lipid peroxidation degradation products in urine of rats treated with carbon tetrachloride using gas chromatography with electron-capture detection, J Chromatogr B Biomed Sci Appl, Vol. 694, No. 2, pp. 277-87.

Ekino, S.; Susa, M.; Ninomiya, T.; Imamura, K. \& Kitamura, T. (2007). Minamata disease revisited: an update on the acute and chronic manifestations of methyl mercury poisoning, J Neurol Sci, Vol. 262, No. 1-2, pp. 131-44.

Eskes, C.; Honegger, P.; Juillerat-Jeanneret, L. \& Monnet-Tschudi, F. (2002). Microglial reaction induced by noncytotoxic methylmercury treatment leads to neuroprotection via interactions with astrocytes and IL-6 release, Glia, Vol. 37, No. 1, pp. 43-52.

Faux, S.P.; Tai, T.; Thorne, D.; Xu, Y.; Breheny, D. \& Gaca, M. (2009). The role of oxidative stress in the biological responses of lung epithelial cells to cigarette smoke, Biomarkers, Vol. 14 Suppl 1, No. 90-6.

Fountoulakis, M.; de Vera, M.C.; Crameri, F.; Boess, F.; Gasser, R.; Albertini, S. \& Suter, L. (2002). Modulation of gene and protein expression by carbon tetrachloride in the rat liver, Toxicol Appl Pharmacol, Vol. 183, No. 1, pp. 71-80.

Gee, D.L.; Bechtold, M.M. \& Tappel, A.L. (1981). Carbon tetrachloride-induced lipid peroxidation: simultaneous in vivo measurements of pentane and chloroform exhaled by the rat, Toxicol Lett, Vol. 8, No. 6, pp. 299-306.

Gieger, C.; Geistlinger, L.; Altmaier, E.; Hrabe de Angelis, M.; Kronenberg, F.; Meitinger, T.; Mewes, H.W.; Wichmann, H.E.; Weinberger, K.M.; Adamski, J.; Illig, T. \& Suhre, K. (2008). Genetics meets metabolomics: a genome-wide association study of metabolite profiles in human serum, PLoS Genet, Vol. 4, No. 11, pp. e1000282.

Ginsberg, G.; Smolenski, S.; Neafsey, P.; Hattis, D.; Walker, K.; Guyton, K.Z.; Johns, D.O. \& Sonawane, B. (2009). The influence of genetic polymorphisms on population variability in six xenobiotic-metabolizing enzymes, J Toxicol Environ Health B Crit Rev, Vol. 12, No. 5-6, pp. 307-33.

Go, E.P. (2010). Database resources in metabolomics: an overview, J Neuroimmune Pharmacol, Vol. 5, No. 1, pp. 18-30.

Guyton, K.Z.; Kyle, A.D.; Aubrecht, J.; Cogliano, V.J.; Eastmond, D.A.; Jackson, M.; Keshava, N.; Sandy, M.S.; Sonawane, B.; Zhang, L.; Waters, M.D. \& Smith, M.T. (2009). Improving prediction of chemical carcinogenicity by considering multiple mechanisms and applying toxicogenomic approaches, Mutat Res, Vol. 681, No. 2-3, pp. 230-40.

Han, J.; Antunes, L.C.; Finlay, B.B. \& Borchers, C.H. (2010). Metabolomics: towards understanding host-microbe interactions, Future Microbiol, Vol. 5, No. 2, pp. 153-61.

Harvey, B.G.; Heguy, A.; Leopold, P.L.; Carolan, B.J.; Ferris, B. \& Crystal, R.G. (2007). Modification of gene expression of the small airway epithelium in response to cigarette smoking, J Mol Med (Berl), Vol. 85, No. 1, pp. 39-53.

Heby, O. (1981). Role of polyamines in the control of cell proliferation and differentiation, Differentiation, Vol. 19, No. 1, pp. 1-20.

Hecht, S.S. (2006). Cigarette smoking: cancer risks, carcinogens, and mechanisms, Langenbecks Arch Surg, Vol. 391, No. 6, pp. 603-13. 
Heijne, W.H.; Kienhuis, A.S.; van Ommen, B.; Stierum, R.H. \& Groten, J.P. (2005). Systems toxicology: applications of toxicogenomics, transcriptomics, proteomics and metabolomics in toxicology, Expert Rev Proteomics, Vol. 2, No. 5, pp. 767-80.

Hu, J.Z.; Rommereim, D.N.; Minard, K.R.; Woodstock, A.; Harrer, B.J.; Wind, R.A.; Phipps, R.P. \& Sime, P.J. (2008). Metabolomics in lung inflammation:a high-resolution (1)h NMR study of mice exposedto silica dust, Toxicol Mech Methods, Vol. 18, No. 5, pp. 385-98.

Huang, X.; Shao, L.; Gong, Y.; Mao, Y.; Liu, C.; Qu, H. \& Cheng, Y. (2008). A metabonomic characterization of $\mathrm{CCl} 4$-induced acute liver failure using partial least square regression based on the GC/MS metabolic profiles of plasma in mice, J Chromatogr B Analyt Technol Biomed Life Sci, Vol. 870, No. 2, pp. 178-85.

Hwang, E.S. \& Kim, G.H. (2007). Biomarkers for oxidative stress status of DNA, lipids, and proteins in vitro and in vivo cancer research, Toxicology, Vol. 229, No. 1-2, pp. 1-10.

Ichi, I.; Kamikawa, C.; Nakagawa, T.; Kobayashi, K.; Kataoka, R.; Nagata, E.; Kitamura, Y.; Nakazaki, C.; Matsura, T. \& Kojo, S. (2009). Neutral sphingomyelinase-induced ceramide accumulation by oxidative stress during carbon tetrachloride intoxication, Toxicology, Vol. 261, No. 1-2, pp. 33-40.

Idle, J.R. \& Gonzalez, F.J. (2007). Metabolomics, Cell Metab, Vol. 6, No. 5, pp. 348-51.

Kelada, S.N.; Eaton, D.L.; Wang, S.S.; Rothman, N.R. \& Khoury, M.J. (2003). The role of genetic polymorphisms in environmental health, Environ Health Perspect, Vol. 111, No. 8, pp. 1055-64.

Kelsen, S.G.; Duan, X.; Ji, R.; Perez, O.; Liu, C. \& Merali, S. (2008). Cigarette smoke induces an unfolded protein response in the human lung: a proteomic approach, $A m J$ Respir Cell Mol Biol, Vol. 38, No. 5, pp. 541-50.

Kim, K.-B.; Chung, M.W.; Um, S.Y.; Oh, J.S.; Kim, S.H.; Na, M.A.; Oh, H.Y.; Cho, W.-S. \& Choi, K.H. (2008). Metabolomics and biomarker discovery: NMR spectral data of urine and hepatotoxicity by carbon tetrachloride, acetaminophen, and Dgalactosamine in rats, Metabolomics, Vol. 4, No. 377-392.

Krewski, D.; Acosta, D., Jr.; Andersen, M.; Anderson, H.; Bailar, J.C., 3rd; Boekelheide, K.; Brent, R.; Charnley, G.; Cheung, V.G.; Green, S., Jr.; Kelsey, K.T.; Kerkvliet, N.I.; Li, A.A.; McCray, L.; Meyer, O.; Patterson, R.D.; Pennie, W.; Scala, R.A.; Solomon, G.M.; Stephens, M.; Yager, J. \& Zeise, L. (2010). Toxicity testing in the 21st century: a vision and a strategy, J Toxicol Environ Health B Crit Rev, Vol. 13, No. 2-4, pp. 51138.

Lin, Y.; Si, D.; Zhang, Z. \& Liu, C. (2009). An integrated metabonomic method for profiling of metabolic changes in carbon tetrachloride induced rat urine, Toxicology, Vol. 256, No. 3, pp. 191-200.

Liu, J.Y.; Tsai, H.J.; Hwang, S.H.; Jones, P.D.; Morisseau, C. \& Hammock, B.D. (2009). Pharmacokinetic optimization of four soluble epoxide hydrolase inhibitors for use in a murine model of inflammation, Br J Pharmacol, Vol. 156, No. 2, pp. 284-96.

Manibusan, M.K.; Odin, M. \& Eastmond, D.A. (2007). Postulated carbon tetrachloride mode of action: a review, J Environ Sci Health Part C, Vol. 25, No. 3, pp. 185-209.

Meng, Q.R.; Gideon, K.M.; Harbo, S.J.; Renne, R.A.; Lee, M.K.; Brys, A.M. \& Jones, R. (2006). Gene expression profiling in lung tissues from mice exposed to cigarette smoke, lipopolysaccharide, or smoke plus lipopolysaccharide by inhalation, Inhal Toxicol, Vol. 18, No. 8, pp. 555-68. 
Monnet-Tschudi, F.; Zurich, M.G. \& Honegger, P. (1996). Comparison of the developmental effects of two mercury compounds on glial cells and neurons in aggregate cultures of rat telencephalon, Brain Res, Vol. 741, No. 1-2, pp. 52-9.

Mortensen, H.M. \& Euling, S.Y. (2011). Integrating mechanistic and polymorphism data to characterize human genetic susceptibility for environmental chemical risk assessment in the 21st century, Toxicol Appl Pharmacol, Vol. No. (In Press).

Myers, G.J.; Thurston, S.W.; Pearson, A.T.; Davidson, P.W.; Cox, C.; Shamlaye, C.F.; Cernichiari, E. \& Clarkson, T.W. (2009). Postnatal exposure to methyl mercury from fish consumption: a review and new data from the Seychelles Child Development Study, Neurotoxicology, Vol. 30, No. 3, pp. 338-49.

NRC (1983). Risk assessment in the federal government: Managing the process, National Academies Press, Washington, DC. Available from: http:/ / www.nap.edu/openbook.php?isbn=0309033497

NRC (1994). Science and judgment in risk assessment, National Academies Press, Washington, DC. Available from: http://www.nap.edu/catalog/2125.html

Pegg, A.E.; Matsui, I.; Seely, J.E.; Pritchard, M.L. \& Poso, H. (1981). Formation of putrescine in rat liver, Med Biol, Vol. 59, No. 5-6, pp. 327-33.

Raamsdonk, L.M.; Teusink, B.; Broadhurst, D.; Zhang, N.; Hayes, A.; Walsh, M.C.; Berden, J.A.; Brindle, K.M.; Kell, D.B.; Rowland, J.J.; Westerhoff, H.V.; van Dam, K. \& Oliver, S.G. (2001). A functional genomics strategy that uses metabolome data to reveal the phenotype of silent mutations, Nat Biotechnol, Vol. 19, No. 1, pp. 45-50.

Rahman, I. \& MacNee, W. (1999). Lung glutathione and oxidative stress: implications in cigarette smoke-induced airway disease, Am J Physiol, Vol. 277, No. 6 Pt 1, pp. L1067-88.

Robertson, D.G. (2005). Metabonomics in toxicology: a review, Toxicol Sci, Vol. 85, No. 2, pp. 809-22.

Robertson, D.G.; Reily, M.D.; Sigler, R.E.; Wells, D.F.; Paterson, D.A. \& Braden, T.K. (2000). Metabonomics: evaluation of nuclear magnetic resonance (NMR) and pattern recognition technology for rapid in vivo screening of liver and kidney toxicants, Toxicol Sci, Vol. 57, No. 2, pp. 326-37.

Roux, A.; Lison, D.; Junot, C. \& Heilier, J.F. (2011). Applications of liquid chromatography coupled to mass spectrometry-based metabolomics in clinical chemistry and toxicology: A review, Clin Biochem, Vol. 44, No. 1, pp. 119-35.

Rubino, F.M.; Pitton, M.; Di Fabio, D. \& Colombi, A. (2009). Toward an "omic" physiopathology of reactive chemicals: thirty years of mass spectrometric study of the protein adducts with endogenous and xenobiotic compounds, Mass Spectrom Rev, Vol. 28, No. 5, pp. 725-84.

Schmelzer, K.R.; Wheelock, A.M.; Dettmer, K.; Morin, D. \& Hammock, B.D. (2006). The role of inflammatory mediators in the synergistic toxicity of ozone and 1-nitronaphthalene in rat airways, Environ Health Perspect, Vol. 114, No. 9, pp. 1354-60.

Serhan, C.N. (2009). Systems approach to inflammation resolution: identification of novel anti-inflammatory and pro-resolving mediators, J Thromb Haemost, Vol. 7 Suppl 1, No. 44-8.

Spira, A.; Beane, J.; Pinto-Plata, V.; Kadar, A.; Liu, G.; Shah, V.; Celli, B. \& Brody, J.S. (2004). Gene expression profiling of human lung tissue from smokers with severe emphysema, Am J Respir Cell Mol Biol, Vol. 31, No. 6, pp. 601-10.

Steiling, K.; Kadar, A.Y.; Bergerat, A.; Flanigon, J.; Sridhar, S.; Shah, V.; Ahmad, Q.R.; Brody, J.S.; Lenburg, M.E.; Steffen, M. \& Spira, A. (2009). Comparison of proteomic and 
transcriptomic profiles in the bronchial airway epithelium of current and never smokers, PLoS One, Vol. 4, No. 4, pp. e5043.

Sun, F.; Hamagawa, E.; Tsutsui, C.; Ono, Y.; Ogiri, Y. \& Kojo, S. (2001). Evaluation of oxidative stress during apoptosis and necrosis caused by carbon tetrachloride in rat liver, Biochim Biophys Acta, Vol. 1535, No. 2, pp. 186-91.

Szeto, S.S.; Reinke, S.N.; Sykes, B.D. \& Lemire, B.D. (2010). Mutations in the Saccharomyces cerevisiae succinate dehydrogenase result in distinct metabolic phenotypes revealed through (1)H NMR-based metabolic footprinting, J Proteome Res, Vol. 9, No. 12, pp. 6729-39.

U.S. EPA (2005). Guidelines for carcinogenic risk assessment, Risk Assessment Forum, U.S. Environmental Proection Agency, Washington, DC, EPA/630/P-03/001F. Available from: http://www.epa.gov/raf/publications/pdfs/CANCER_GUIDELINES_FINAL_325-05.PDF

U.S. EPA (1992). Respiratory health effects of passive smoking: lung cancer and other disorders, U.S. Environmental Protection Agency, Washington, DC, EPA/600/690/006F. Available from: http://www.epa.gov/ncea/ets/pdfs/acknowl.pdf

U.S. EPA (2001). Water Quality Criterion for the Protection of Human Health: Methylmercury, U.S. Environmental Protection Agency, Washington, DC, EPA823-R-01-001. Available from:

http://water.epa.gov/scitech/swguidance/standards/criteria/aqlife/pollutants/ methylmercury/upload/2009_01_15_criteria_methylmercury_mercurycriterion.pdf

van Ravenzwaay, B.; Cunha, G.C.; Leibold, E.; Looser, R.; Mellert, W.; Prokoudine, A.; Walk, T. \& Wiemer, J. (2007). The use of metabolomics for the discovery of new biomarkers of effect, Toxicol Lett, Vol. 172, No. 1-2, pp. 21-8.

van Vliet, E.; Morath, S.; Eskes, C.; Linge, J.; Rappsilber, J.; Honegger, P.; Hartung, T. \& Coecke, S. (2008). A novel in vitro metabolomics approach for neurotoxicity testing, proof of principle for methyl mercury chloride and caffeine, Neurotoxicology, Vol. 29, No. 1, pp. 1-12.

Vulimiri, S.V.; Berger, A. \& Sonawane, B. (2011). The potential of metabolomic approaches for investigating mode(s) of action of xenobiotics: case study with carbon tetrachloride, Mutat Res, Vol. 722, No. 2, pp. 147-53.

Vulimiri, S.V.; Misra, M.; Hamm, J.T.; Mitchell, M. \& Berger, A. (2009). Effects of mainstream cigarette smoke on the global metabolome of human lung epithelial cells, Chem Res Toxicol, Vol. 22, No. 3, pp. 492-503.

Wang, C.; Yang, J. \& Nie, J. (2009). Plasma phospholipid metabolic profiling and biomarkers of rats following radiation exposure based on liquid chromatography-mass spectrometry technique, Biomed Chromatogr, Vol. 23, No. 10, pp. 1079-85.

Waters, N.J.; Waterfield, C.J.; Farrant, R.D.; Holmes, E. \& Nicholson, J.K. (2006). Integrated metabonomic analysis of bromobenzene-induced hepatotoxicity: novel induction of 5-oxoprolinosis, J Proteome Res, Vol. 5, No. 6, pp. 1448-59.

West, P.R.; Weir, A.M.; Smith, A.M.; Donley, E.L. \& Cezar, G.G. (2010). Predicting human developmental toxicity of pharmaceuticals using human embryonic stem cells and metabolomics, Toxicol Appl Pharmacol, Vol. 247, No. 1, pp. 18-27.

Zhang, S.; Xu, N.; Nie, J.; Dong, L.; Li, J. \& Tong, J. (2008). Proteomic alteration in lung tissue of rats exposed to cigarette smoke, Toxicol Lett, Vol. 178, No. 3, pp. 191-6. 


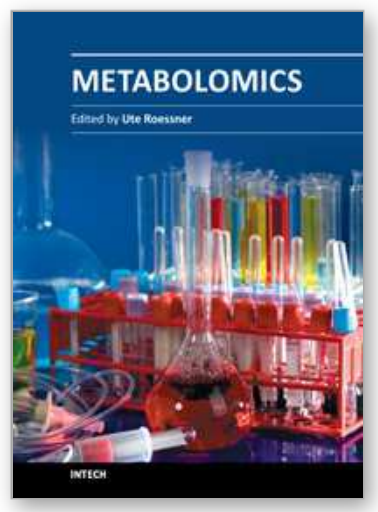

\author{
Metabolomics \\ Edited by Dr Ute Roessner
}

ISBN 978-953-51-0046-1

Hard cover, 364 pages

Publisher InTech

Published online 10, February, 2012

Published in print edition February, 2012

Metabolomics is a rapidly emerging field in life sciences, which aims to identify and quantify metabolites in a biological system. Analytical chemistry is combined with sophisticated informatics and statistics tools to determine and understand metabolic changes upon genetic or environmental perturbations. Together with other 'omics analyses, such as genomics and proteomics, metabolomics plays an important role in functional genomics and systems biology studies in any biological science. This book will provide the reader with summaries of the state-of-the-art of technologies and methodologies, especially in the data analysis and interpretation approaches, as well as give insights into exciting applications of metabolomics in human health studies, safety assessments, and plant and microbial research.

\title{
How to reference
}

In order to correctly reference this scholarly work, feel free to copy and paste the following:

Suryanarayana V. Vulimiri, Brian Pachkowski, Ambuja S. Bale and Babasaheb Sonawane (2012). Metabolomics Approach for Hazard Identification in Human Health Assessment of Environmental Chemicals, Metabolomics, Dr Ute Roessner (Ed.), ISBN: 978-953-51-0046-1, InTech, Available from: http://www.intechopen.com/books/metabolomics/metabolomics-approach-for-hazard-identification-in-humanhealth-assessment-of-environmental-chemical

\section{INTECH}

open science | open minds

\author{
InTech Europe \\ University Campus STeP Ri \\ Slavka Krautzeka 83/A \\ 51000 Rijeka, Croatia \\ Phone: +385 (51) 770447 \\ Fax: +385 (51) 686166 \\ www.intechopen.com
}

\author{
InTech China \\ Unit 405, Office Block, Hotel Equatorial Shanghai \\ No.65, Yan An Road (West), Shanghai, 200040, China \\ 中国上海市延安西路65号上海国际贵都大饭店办公楼 405 单元 \\ Phone: +86-21-62489820 \\ Fax: +86-21-62489821
}


(C) 2012 The Author(s). Licensee IntechOpen. This is an open access article distributed under the terms of the Creative Commons Attribution 3.0 License, which permits unrestricted use, distribution, and reproduction in any medium, provided the original work is properly cited. 